Editorial

\section{Metabolomics in asthma}

\section{Editorial}

Metabolomics is the quantitative study of sets of small molecules with molecular masses less than 1 kilo Dalton $(\mathrm{kDa})$ generated from cellular metabolic activity. A large number of metabolites that result from genomic, transcriptomic, proteomic, and environmental variability can be assessed by current technologies. These metabolomics data therefore are valuable to provide the most integrated profiles of the complex interactions of genes and environment, including disease predisposing, diagnosis, and disease progression. Currently, mass spectrometry (MS) coupled with liquid chromatography (LC) is the most availably advanced technology. This technique can be used both in a targeted or non-targeted, pattern-recognition manner. By using genetic, environmental, genomic, and clinical data in combination with descriptive metabolic profiles obtained by MS, It is possible to identify biomarkers and patterns of changes that differentiate between states asthma severity and asthmatic cases and controls. To date, research works in asthma metabolomics has been limited, in contrast to cardiovascular disease, Alzheimer's disease, and type2 diabetes with the discovery of their novel disease pathways. The severe asthmatic with high fraction of exhaled nitric oxide (FENO) has unique endotypes that indicates change in bile acid metabolism and NO-associated taurine transport. Sera of asthmatic patients are characteristically demonstrated by decreased levels of glucose, arginine, O-phosphocholine, choline, acetate, methanol, and formate, and increased levels of histidine, glutamine, and methionine. The serum metabolites in patients with asthma are involved in immune reaction, response to hypoxia, and hypermethylation. Additionally, the levels of these serum metabolites associate with asthma severity. Particularly, lipid metabolism is changed in patients with lower forcedexpiratory volume in one second percentage $\left(\mathrm{FEV}_{1} \%\right)$ predicted values. A previous study demonstrated strong predictive power of potential biomarkers in ROC analysis. The presence of asthma in external validation models was predicted with high accuracy $(100 \%$ for controls and $90.9 \%$ for asthmatic patients). In conclusions, several published studies on the metabolomics of asthma are limited in scope and number. Most of these studies demonstrated good predictive
Volume 4 Issue I - 2017

\author{
Attapon Cheepsattayakorn, ${ }^{1,2,3}$ Ruangrong \\ Cheepsattayakorn ${ }^{4}$ \\ 'Editor-in-Chief, Journal of Lung, Pulmonary and Respiratory \\ Research, USA \\ ${ }^{2}$ I Oth Zonal Tuberculosis and Chest Disease Center, Chiang Mai, \\ Thailand \\ ${ }^{3} 5$ th Office of Disease Prevention and Control, Ratchaburi, \\ Department of Disease Control, Ministry of Public Health, \\ Thailand \\ ${ }^{4}$ Department of Pathology, Faculty of Medicine, Chiang Mai \\ University, Chiang Mai, Thailand
}

Correspondence: Attapon Cheepsattayakorn, I0th Zonal Tuberculosis and Chest Disease Center, 143 Sridornchai Road Changklan Muang Chiang Mai 50I00 Thailand, Tel 6653 I40767, 6653276364 Fax 6653 140773, 6653 273590,

Email Attapon 1958@gmail.com, attaponche@yahoo.com

Received: February 24, 2017 | Published: February 28, 2017

accuracy in differentiating controls from asthmatics, but they are limited in the number of metabolites, size, and the complexity of phenotype(s). Additionally, these studies lack replication. The need of large, well characterized asthmatic cohorts for both validating and discovering asthma metabolomics studies is critical to be performed.

\section{Acknowledgements}

None.

\section{Conflict of interest}

The author declares no conflict of interest. 